\title{
Behavioral expression of learned fear in rats is appropriate to their age at training, not their age at testing
}

\author{
RICK RICHARDSON and MICHELLE FAN \\ University of New South Wales, Sydney, New South Wales, Australia
}

\begin{abstract}
Recent research has shown that learned fear emerges in a response-specific sequence. For example, an odor conditioned stimulus (CS) previously paired with shock elicits behavioral expressions of fear like avoidance at a younger age than it elicits other behavioral expressions of fear like potentiation of the startle response (Richardson, Paxinos, \& Lee, 2000). In the present study, the question of whether learned fear is expressed in a manner appropriate to the animal's age at training or its age at testing was explored in three experiments, all using a within-subjects design. The results suggest that learned fear is expressed in a manner appropriate to the rat's age at training, not its age at testing. The Discussion section focuses on the implications of these findings for (1) the developmental analysis of memory and (2) the idea that an aversive CS elicits a central state of fear.
\end{abstract}

Recent research on the developing rat has shown that learned fear emerges in a response-specific sequence. That is, three common measures of learned fear in the rat are freezing (e.g., Fanselow, 1990; Westbrook, Good, \& Kiernan, 1994), changes in autonomic function (i.e., heart rate or blood pressure; e.g., Carrive, 2000; Hunt, Richardson, Hess, \& Campbell, 1997), and a potentiated startle response to a loud, unexpected noise (McNish, Gewirtz, \& Davis, 1997; Vishney \& Richardson, 2000). When a tone is used as the conditioned stimulus (CS), conditioned freezing emerges at a younger age than does conditioned changes in heart rate, which emerges before conditioned fear potentiation of startle (for a review, see Hunt \& Campbell, 1997). This same pattern occurs with a visual CS (e.g., Hunt, Richardson, \& Campbell, 1994). Learned fear emerges in a response-specific sequence with olfactory CSs as well. For example, an aversive odor CS elicits avoidance responses (Rudy \& Cheatle, 1977) at younger ages than it elicits changes in heart rate (Sananes, Gaddy, $\&$ Campbell, 1988). We have recently reported additional evidence showing the sequential emergence of learned fear to an olfactory CS; specifically, conditioned odorelicited freezing emerges at a younger age than does conditioned odor potentiation of startle (OPS; Richardson, Tronson, Bailey, \& Parnas, 2002).

This research was submitted by M.F. in partial fulfillment of the requirements for an honors degree at UNSW. The research was supported by Grant A79800074 from the Australian Research Council and a University Research Support Program grant from UNSW to R.R. The authors acknowledge the helpful feedback of Marianne Weber on an earlier draft of the manuscript. Correspondence concerning this article may be sent to R. Richardson, School of Psychology, The University of New South Wales, Sydney 2052, Australia (e-mail: r.richardson@unsw. edu.au).
This sequential emergence of learned fear provides a framework for exploring a fundamental question concerning the development of memory. Specifically, do animals express their memories in a manner appropriate to their age at training or their age at testing? In other words, what happens if rats are trained at an age at which they can express learned fear via one response, but not another, and are then tested at an age at which they can express fear via both responses? We recently reported data relevant to this issue (Richardson, Paxinos, \& Lee, 2000). In that study, we first showed that an odor previously paired with shock potentiated the acoustic startle response in rats trained at 23 days of age or older. This same odor CS was ineffective in potentiating the startle response in rats trained at 16 or 20 days of age. However, the failure to observe conditioned OPS in rats younger than 23 days of age cannot be attributed to an inability to either detect or learn about the odor or to the forgetting of the odor-shock association over the 24-h retention interval, because the odor CS elicited a pronounced avoidance in the 16-day-olds. The most surprising finding of that study, however, was that rats trained at 16 days of age but not tested until 23 days of age failed to exhibit the OPS effect, even though they exhibited complete retention of the CS-unconditioned stimulus (US) association over the 1-week retention interval (as shown by the level of odor avoidance). In other words, these rats responded at test in a manner appropriate to their age at training (i.e., they avoided the odor but did not exhibit OPS).

This finding was completely unexpected. We had predicted that these rats would exhibit conditioned OPS. The basis for this prediction was the generally accepted view that an aversive CS elicits a central state of learned fear (e.g., Davis, 1992) - a state that can be expressed via any of a number of responses. Therefore, if the animals re- 
member the CS-US association over the retention interval (which they do), the central state of fear elicited by the CS should be expressed via any response system that is functional at the time of testing. That clearly was not what was found. Because of the potential significance of these findings for (1) the developmental analysis of memory and (2) the idea that an aversive CS elicits a central state of fear, we systematically explored this issue in the present study.

\section{GENERAL METHOD}

\begin{abstract}
Subjects
Experimentally naive Sprague Dawley rats, obtained from the breeding colony maintained by the School of Psychology at the University of New South Wales, were used. Animals were either 16 $( \pm 1)$ or $23( \pm 1)$ days of age at training. Rats of both sexes were used, and they were housed in litters of 8 , with their mothers, in plastic boxes $(37 \times 24.5 \times 27 \mathrm{~cm}$, length $\times$ width $\times$ height $)$ kept in a room with a 12:12-h light:dark cycle (lights on at 6 a.m.). No more than 1 subject from any single litter was included in a group. Food and water were continuously available. Training and testing occurred between 9 a.m. and 5 p.m. All the animals were treated in accord with the Australian code of practice for the care and use of animals for scientif ic purposes, and the Animal Care and Ethics Committee at the University of New South Wales approved all procedures.
\end{abstract}

\section{Apparatus}

Two types of chambers were used in this study: startle chambers and an odor avoidance chamber.

Startle chambers. To assess startle, rats were tested in one of two rectangular chambers constructed of Plexiglas and stainless steel bars. The front wall, rear wall, and ceiling of each startle chamber were constructed of clear Plexiglas. The floor and two sidewalls were constructed of 3-mm stainless steel rods (1 cm apart, center to center; the wall rods were vertically positioned relative to the floor rods). Electric shock ( $0.6 \mathrm{~mA}, 1 \mathrm{sec}$ in duration) could be delivered to the floor of each chamber via a custom-built, constant-current shock generator. The chamber was attached to a piece of Plexiglas onto which a sheet of piezoelectric film had been laminated. Movement within the chamber caused the piece of Plexiglas to flex, which produced a voltage in the piezoelectric film. The voltage produced by the piezoelectric film was proportional to the intensity of the movement in the chamber; that is, larger movements produced larger voltages. These voltages were amplified and digitized (at a 1-kHz rate) in order to measure startle amplitude. The peak voltage (converted into arbitrary units ranging from 0 to 32,000 , rounded to $0-320$ prior to analysis) in the 250 -msec period after stimulus onset was taken as the index of the startle response.

Each chamber was located in a wood cabinet in order to attenuate external noise and visual stimulation. A ventilation fan in the cabinet provided a low-level background noise $(60 \mathrm{~dB})$ at all times, and illumination was provided by a $15-\mathrm{W}$ red light on the front door of the wood cabinet. Following each training or test session, the startle chambers were cleaned with tap water, and a tray of animal bedding just below each chamber was changed.

The acoustic startle stimulus was delivered through two highfrequency speakers mounted $8 \mathrm{~cm}$ from either side of the startle chamber. The startle stimulus was a $100-\mathrm{msec}, 100-\mathrm{dB}$ burst of white noise, with a 1-msec rise-fall time. The intensity of the startle stimulus and of the background noise was measured with a Brüel and Kjaer precision sound level meter (Type 2235) placed in the center of the startle cage. All stimulus presentations and recording of data were controlled by computer. The software and hardware were custom developed at the University of New South Wales.
Avoidance chamber. A rectangular chamber $(29 \times 15 \times 9.5 \mathrm{~cm}$, length $\times$ width $\times$ height) made of Plexiglas was placed on a grid floor consisting of stainless steel grids $(3 \mathrm{~mm}$ in diameter, $0.7 \mathrm{~cm}$ apart, center to center). The chamber was divided into three sections. The middle $4.5 \mathrm{~cm}$ of the chamber was termed the neutral zone, one end was termed the odor zone, and the other end was termed the noodor zone (each end was $12.25 \mathrm{~cm}$ long). A specimen jar containing the grape odorant (see below) was placed under the odor zone. The times spent in both the odor and the no-odor zones during a 3-min test were recorded. A rat was considered to be out of the neutral zone whenever its head left that area of the chamber.

Odor. The odor used in this study was $0.1 \mathrm{~mL}$ of grape flavor (Grape No. 182380019 from Wild Flavours, Heidelberg, Germany); in the control condition, $0.1 \mathrm{~mL}$ of tap water was used in place of the grape. In both cases, the fluid (either water or grape) was squirted onto a piece of paper towel inside the specimen jar. During odor presentation, the jar was placed approximately $10 \mathrm{~cm}$ below the startle or avoidance chamber floor.

\section{Procedure}

Training. A two-stage training procedure was used in all the experiments, and training always occurred in a startle chamber. The doors of the wood cabinets in which the startle cages were located were kept slightly ajar throughout both stages of training. In Stage 1 of training, the rats were placed in the startle chamber and given a 5-min adaptation period, at the end of which the first of 15 shocks was given. Seven to $10 \mathrm{sec}$ prior to each of these shocks, a jar (containing grape for the rats in the paired group and water for the rats in the unpaired group) was placed beneath the startle chamber. Immediately after the shock, the jar was removed, covered with a lid, and placed on a bench approximately $2 \mathrm{~m}$ from the startle chamber. The interval between shock presentations was $1.5,2$, or $2.5 \mathrm{~min}$ (average $=2 \mathrm{~min}$ ) and varied pseudorandomly. After the last shock, the rats were removed from the startle chambers and placed in their home cages. This training procedure produces an association between grape and shock in the rats in the paired condition, but not in the rats in the unpaired condition. The rats in both conditions, however, would acquire an association between the context (i.e., the startle cage) and shock. Therefore, in order to extinguish this contextshock association, at least partially, and to equate the exposure to the grape odor for the rats in the paired and the unpaired conditions, a second stage of training was given. In Stage 2 of training, the rats were returned to the startle chamber that they had been in during Stage 1 and were given a 2-min adaptation period. After this, a jar, containing water for rats in the paired condition and grape for rats in the unpaired condition, was presented for 7-10 sec. No shocks were administered in Stage 2 of training. The jar was presented 15 times in Stage 2 (i.e., the same number of times as in Stage 1), and the interval between presentations was $1.5,2$, or $2.5 \mathrm{~min}$ (average $=2$ min) and varied pseudorandomly (i.e., the same as in Stage 1). Both stages of training lasted approximately $35 \mathrm{~min}$, and the interval between the two stages was 50-60 min. At the end of each stage of training, the doors and windows were opened for at least $10 \mathrm{~min}$ to allow adequate ventilation of the room.

Testing. There were two types of test procedures used in this study. All the rats received both tests in a counterbalanced order (because subsequent statistical analysis showed that test order did not influence the results in any experiment, all the data were collapsed across this variable). The two tests were separated by at least $3 \mathrm{~h}$. Tests occurred either 1 or 7 days after training in Experiment 1, 1 day after training in Experiment 2, and 7 days after training in Experiment 3.

In the startle test, the rats were placed in a startle chamber, and after a 5-min adaptation period, 30 startle-eliciting noise bursts, separated by $30 \mathrm{sec}$, were presented. No odor was present during these initial 30 bursts. The average startle response on these initial 30 trials was taken as an estimate of the rat's baseline startle response. 
Table 1

Mean $( \pm S E M)$ Baseline Startle Response in 16- and 23-Day-Old Rats in Experiment 1

\begin{tabular}{lcrlrr}
\hline & \multicolumn{2}{c}{ 16-Day-Olds } & & \multicolumn{2}{c}{ 23-Day-Olds } \\
\cline { 2 - 3 } \cline { 6 - 6 } \multicolumn{1}{c}{ Group } & $M$ & $S E M$ & & $M$ & $S E M$ \\
\hline Paired-1-Day & 19.6 & 3.5 & & 34.9 & 9.5 \\
Paired-7-Day & 53.1 & 20.2 & & 37.9 & 8.8 \\
Unpaired-1-Day & 32.4 & 12.3 & & 43.0 & 7.2 \\
Unpaired-7-Day & 31.3 & 7.9 & & 58.5 & 12.3 \\
\hline
\end{tabular}

During a 60 -sec period following the 30th noise burst, a jar containing grape was placed beneath the startle chamber floor and remained there for the rest of the test session (about $15 \mathrm{~min}$ ). After the introduction of the grape odor, an additional 30 noise bursts, each separated by $30 \mathrm{sec}$, were presented. At the end of the testing session, the subjects were removed from the startle chamber and were returned to their home cages.

OPS was examined by comparing the average startle amplitude when the odor was present with the average startle amplitude during baseline and was calculated using the following equation: [ $T-$ $B) / B] \times 100=$ OPS, where $T$ is the mean startle amplitude over the 30 test trials, $B$ is the mean startle amplitude over the 30 baseline trials, and OPS is the percentage increase in startle.

For the odor avoidance test, the rats were placed in the neutral zone of the avoidance chamber, described above, for a single 3-min test session. A rat was considered to be out of the neutral zone if its head was within either the odor or the no-odor zone. Odor avoidance was determined by dividing the time spent in the odor zone by the time spent in the odor and no-odor zones (i.e., time in the neutral zone was excluded). All time measures were made with standard stopwatches.

\section{EXPERIMENT 1}

As was noted earlier, previous research from our laboratory had shown that rats given odor-shock pairings at
16 days of age exhibited odor avoidance, but not OPS, whereas rats trained at 23 days of age exhibited both forms of learned fear (Richardson et al., 2000). Furthermore, the results of that study suggested that rats trained at 16 days of age but not tested until 23 days of age perform in a manner appropriate to their age at training (i.e., they exhibit conditioned odor avoidance, but not OPS). Because of the potential impact of these findings and because our earlier study relied on making cross-experiment comparisons, we designed the present experiment to more systematically examine the issue of whether rats express learned fear in a manner appropriate to their age at the time of training or their age at the time of testing.

\section{Method}

\section{Subjects}

Sixty-four experimentally naive Sprague Dawley-derived rats were used. The subjects were either 16 or 23 days of age at training. At each age, the rats were randomly assigned to one of four groups: Paired-1-Day, Paired-7-Day, Unpaired-1-Day, or Unpaired-7-Day (the first label refers to whether the grape odor was paired with shock or not, and the second label indicates the interval between training and test). The data from one 16-day-old rat (in Group Unpaired-1-Day) was excluded from the statistical analysis because it failed to leave the neutral zone during the odor avoidance test (because this experiment focused on a within-subjects comparison, the startle data of this subject were excluded as well). Therefore, all the groups contained 8 subjects, except for the 16-day-old Unpaired-1Day group, which contained 7 subjects.

\section{Results and Discussion}

\section{Startle}

Baseline. The mean $( \pm S E M)$ baseline startle amplitude for each group is presented in Table 1 . There were no significant effects or interactions revealed in a $2 \times 2 \times 2$

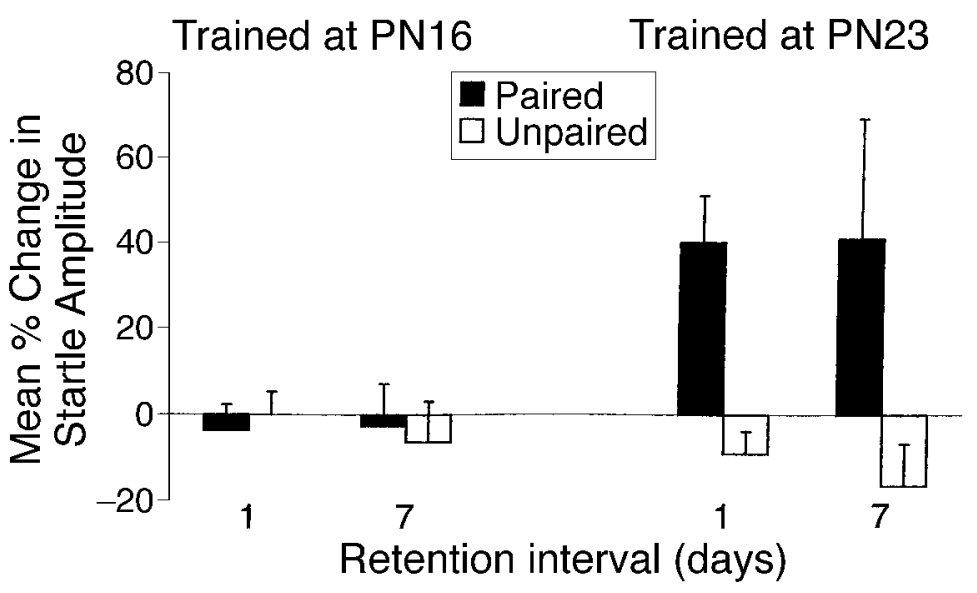

Figure 1. Mean $( \pm S E M)$ percentage change in startle amplitude during presentation of grape odor. The rats were either 16 or 23 days of age at the time of training and were tested either 1 or 7 days after training. The rats in the paired condition (filled bars) received 15 pairings of grape and shock at training, whereas the rats in the unpaired condition (open bars) received the same number of grape and shock presentations, but in an explicitly unpaired fashion. 
analysis of variance (ANOVA; age $\times$ condition $\times$ retention interval) of baseline startle amplitudes [largest $F(1,55)=$ $2.58, p=.11$, for the retention interval effect].

Test. The mean ( $\pm S E M)$ percentage change from baseline startle amplitude during the odor test is presented in Figure 1. An inspection of this figure suggests that the rats given paired presentations of the odor and shock at 23 days of age exhibited a significant OPS effect, regardless of the retention interval. In contrast, the rats given paired presentations of the odor and shock at 16 days of age did not exhibit an OPS effect at either retention interval. A statistical analysis confirmed these interpretations. Specifically, analysis of the startle test data revealed a significant effect of condition $[F(1,55)=8.65, p=.005]$ and a significant age $\times$ condition interaction $[F(1,55)=8.47, p=$ $.005]$. No other main effects or interactions were significant [largest $F(1,55)=3.55, p=.065$, for the main effect of age]. The significant age $\times$ condition interaction was due to the significant difference between the paired and the unpaired rats trained at 23 days of age $[t(30)=3.41, p=$ $.003]$, but not for those trained at 16 days of age $[t(29)=$ $0.04, p=.96]$.

\section{Odor Avoidance}

The mean $( \pm S E M)$ percentage of time spent in the odor zone is depicted in Figure 2. An inspection of this figure suggests that the rats given odor-shock pairings, at either 16 or 23 days of age, avoided the odor, as compared with the rats given the odor and the shock in an explicitly unpaired fashion. This effect occurred whether the rats were tested after a 1- or a 7-day retention interval. Finally, the rats trained at 23 days of age appeared to avoid the odor more than did the rats trained at 16 days of age. A statistical analysis confirmed these interpretations of the data. Specifically, an analysis of the odor avoidance test data re- vealed a significant effect of condition $[F(1,55)=61.29$, $p<.001]$ and age $[F(1,55)=5.14, p=.027]$ but no effect of retention interval $(F<1.0)$ or any significant interactions [largest $F(1,55)=1.17$ ]. The significant main effect of condition was due to the paired rats' spending less time in the odor zone than did the unpaired rats $[t(29)=$ 5.35 and $t(30)=5.79$, both $p s<.001$, for the 16- and the 23-day-olds, respectively]. The significant main effect of age was due to a stronger avoidance of the odor being shown by the rats trained at 23 days of age than by those trained at 16 days of age; post hoc analyses showed that this age difference was significant for the rats in the paired condition $[t(30)=3.52, p=.001]$, but not for those in the unpaired condition $[t(29)=1.30, p=.20]$.

The results of this experiment replicate and extend previous findings from our laboratory. That is, rats trained at 16 days of age fail to exhibit the OPS effect, whereas rats trained at 23 days of age do exhibit the OPS effect (Richardson et al., 2000). The failure to observe OPS in the 16day-old rats is, importantly, not due to a failure of these rats in acquiring the odor-shock association. That is, these rats do avoid the odor. This dissociation was made particularly striking in the present experiment because of the within-subjects design that was used.

The results of this experiment also show that rats trained at 16 days of age but not tested until 23 days did not exhibit the OPS effect, even though they retained the odor-shock association over the 1-week interval. This finding is not what would be expected from the perspective that an aversive CS elicits a central state of fear. That is, these rats were tested at an age at which they could exhibit the OPS effect, and even though they clearly retained the odor-shock association, they did not exhibit OPS. However, these results are in agreement with our earlier, preliminary findings (Richardson et al., 2000) and pro-

\section{Trained at PN16}

\section{Trained at PN23}

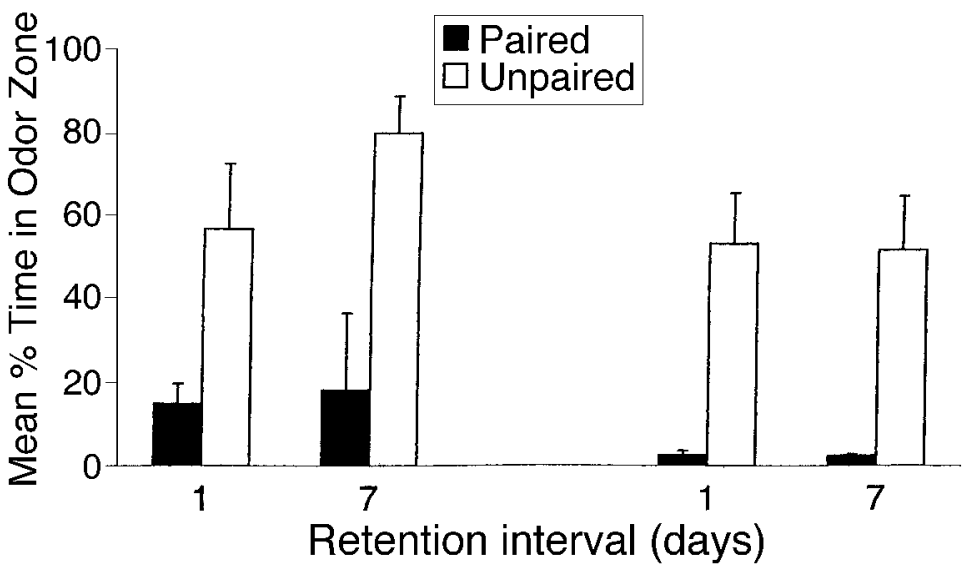

Figure 2. Mean $( \pm S E M)$ percentage of time in the odor zone during a 3-min test. The rats were either 16 or 23 days of age at the time of training and were tested either 1 or 7 days after training. The rats in the paired condition (filled bars) received 15 pairings of grape and shock at training, whereas the rats in the unpaired condition (open bars) received the same number of grape and shock presentations, but in an explicitly unpaired fashion. 
vide strong support for the conclusion that rats express learned fear in a manner appropriate to their age at training, rather than their age at testing.

\section{EXPERIMENT 2}

Although the results of Experiment 1 provide strong support for our earlier conclusion that rats express learned fear in a manner appropriate to their age at training, and not their age at testing, one aspect of the results raises a potential problem for our past analysis of the sequential emergence of learned fear to an odor CS. Specifically, we reported that an odor CS was capable of eliciting odor avoidance or freezing at a younger age than that at which it was capable of eliciting OPS (Richardson et al., 2000; Richardson et al., 2002). Our interpretation of that finding was that the neural circuit responsible for fear potentiation of startle is not functional in the 16-day-old rat but is functional in the 23-day-old rat (see, also, Hunt \& Campbell, 1997). However, the results of the odor avoidance test in Experiment 1 suggest a very different potential interpretation of the observed developmental dissociation of these various forms of learned fear.

In our previous study on the ontogeny of conditioned OPS, we tested some 16-day-old rats on an odor avoidance procedure in order to ensure that the observed lack of the OPS effect was not due to a failure to acquire, or remember, the odor-shock association in rats of this age (Richardson et al., 2000). Given that rats trained at 23 days of age expressed the OPS effect, we did not test rats this age on the odor avoidance procedure in that earlier work. However, in the present study, we tested both 16- and 23day-old rats on the odor avoidance procedure. The results showed that rats of both ages given odor-shock pairings subsequently avoided that odor but, more important, that rats trained at 23 days of age exhibited a stronger conditioned avoidance of the odor than did rats trained at 16 days of age. This finding could provide the basis for a threshold account of the failure to observe the OPS effect in rats trained at 16 days of age. That is, the level of fear required for avoidance may be less than that required for potentiation of startle, and because 16-day-old rats exhibit a slightly weaker avoidance of the odor CS than do 23-dayold rats, it might be the case that the level of fear elicited in the younger rats is not sufficient for producing conditioned fear potentiation of startle. In the present experiment, this hypothesis was tested directly.

The idea that the level of fear elicited by the odor CS is sufficient to produce avoidance at both ages but exceeds the threshold for producing fear potentiation of startle only at 23 days of age, can be directly examined in either of two ways. First, one could increase the number of odor-shock pairings for the 16-day-old rats in an effort to increase their level of odor avoidance to that observed in the 23-day-olds. Second, one could reduce the number of odor-shock pairings in the 23-day-olds in an effort to reduce their level of odor avoidance to that observed in the 16-day-olds. If the threshold notion is correct, we would observe the OPS effect in both age groups in the first case and in neither age group in the second case. In the present experiment, we used the second scenario to explicitly test whether the observed age differences in how learned fear is expressed is merely a matter of different thresholds for conditioned odor avoidance and fear potentiation of startle.

\section{Method}

\section{Subjects}

Forty experimentally naive Sprague Dawley-derived rats, obtained from the same source and housed in the same manner as those in Experiment 1 , were used. The subjects were either 16 or 23 days of age at training.

\section{Procedure}

At each age, the rats were randomly assigned to one of two groups: paired or unpaired. The rats in the paired group received multiple pairings of the grape odor and shock, whereas the rats in the unpaired group received an equivalent number of grape and shock presentations, but in an explicitly unpaired fashion (see the General Method section). The rats trained at 16 days of age received 15 odorshock presentations, whereas the rats trained at 23 days of age received only 5 odor-shock presentations. All the rats were tested (on the startle and odor avoidance tests) 1 day after training. All the groups contained 10 subjects.

\section{Results and Discussion}

\section{Startle}

Baseline. The mean $( \pm S E M)$ baseline startle amplitude for each group is presented in Table 2. In this experiment, there was a significant effect of age on baseline startle amplitude $[F(1,36)=18.7, p<.001]$. The effect of condition and the age $\times$ condition interaction were not significant (both $F_{\mathrm{s}}<1.0$ ). The main effect of age was due to the 23day-old rats' having a higher baseline startle amplitude than did the 16-day-olds. This difference did not necessarily reflect a true age difference in startle magnitude. That is, we varied the gain setting for each startle chamber for rats of different ages in an attempt to produce comparable baselines. Obviously, we were unsuccessful in this effort in this particular experiment. In any case, given that we converted each animal's test score into a percentage change from the baseline score, the effects of the age differences in baseline startle amplitude were minimized. Furthermore, if anything, the high baselines in the 23-day-olds reduced the likelihood of observing the OPS effect in rats this age, and the low baselines in the 16-day-olds maximized the likelihood of observing the OPS effect in rats this age.

Test. The mean $( \pm S E M)$ percentage change from baseline startle amplitude during the odor test is presented in Figure 3 . An inspection of this figure suggests that the rats

Table 2

Mean $( \pm S E M)$ Baseline Startle Response in 16- and 23-Day-Old Rats in Experiment 2

\begin{tabular}{lccccc}
\hline & \multicolumn{2}{c}{ 16-Day-Olds } & & \multicolumn{2}{c}{ 23-Day-Olds } \\
\cline { 2 - 3 } \cline { 5 - 6 } Condition & $M$ & $S E M$ & & $M$ & $S E M$ \\
\hline Paired & 27.6 & 5.2 & & 56.4 & 7.6 \\
Unpaired & 31.7 & 7.6 & 63.0 & 7.2 \\
\hline
\end{tabular}




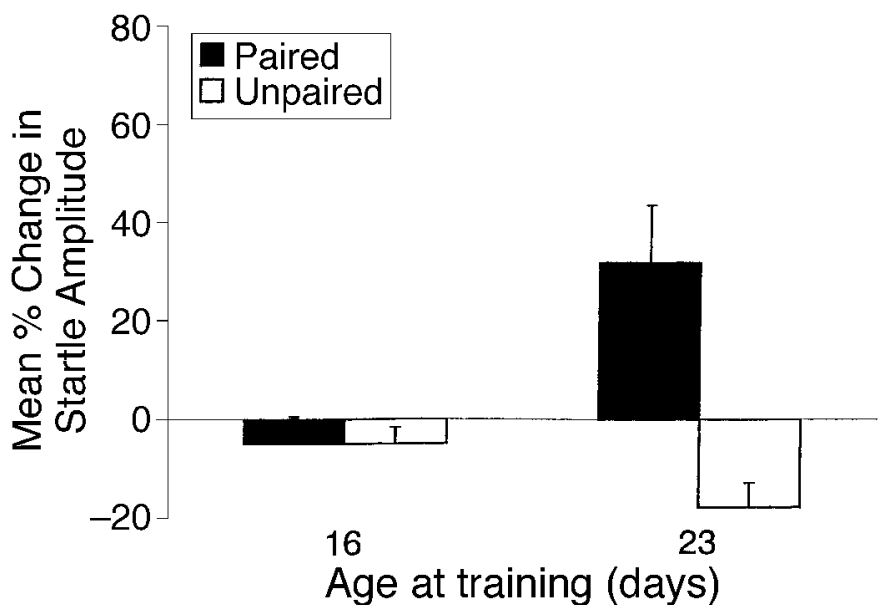

Figure 3. Mean $( \pm S E M)$ percentage change in startle amplitude during presentation of grape odor. The rats were either 16 or 23 days of age at the time of training and were tested 1 day after training. The rats in the paired condition (filled bars) received either 5 or 15 pairings of grape and shock at training (the 23 - and 16-day-old rats, respectively), whereas the rats in the unpaired condition (open bars) received the same number of grape and shock presentations, but in an explicitly unpaired fashion.

given paired presentations of the odor and the shock at 23 days of age exhibited a significant OPS effect, in comparison with the rats given unpaired presentations of the odor and the shock. In contrast, the rats given paired presentations of the odor and the shock at 16 days of age did not exhibit an OPS effect. A statistical analysis confirmed these interpretations. Specifically, an analysis of the star- tle test data yielded significant main effects of age and condition [smallest $F(1,36)=18.4, p<.001$ ], as well as a significant age $\times$ conditioninteraction $[F(1,36)=18.1, p=$ $.001]$. The significant age $\times$ conditioninteraction was due to the paired rats' exhibiting an OPS effect as compared with the unpaired rats at 23 days of age $[t(18)=5.05, p<$ $.001]$, but not at 16 days of age $[t(18)=0.48, p=.63]$.

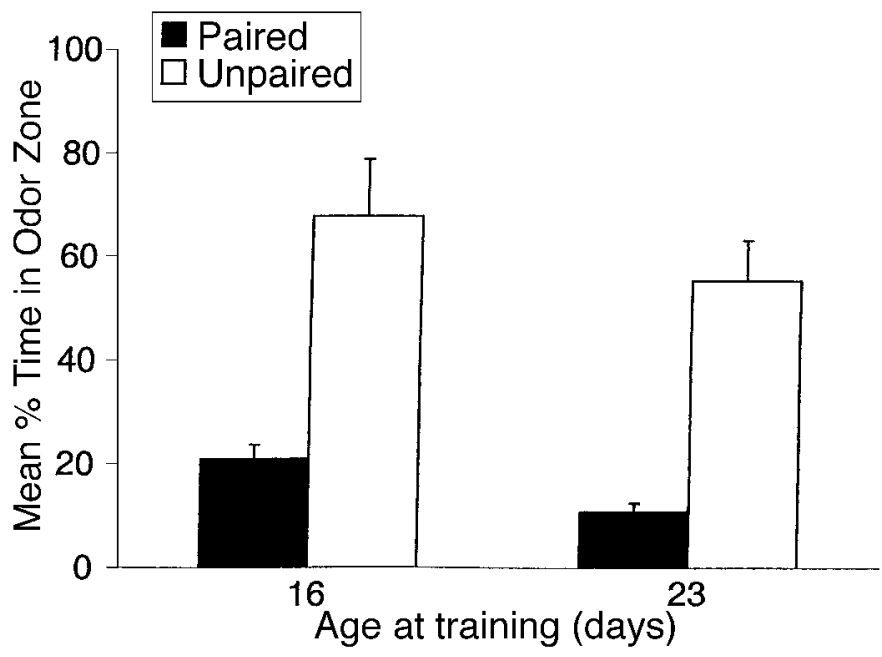

Figure 4. Mean $( \pm S E M)$ percentage of time in the odor zone during a 3-min test. The rats were either 16 or 23 days of age at the time of training and were tested 1 day after training. The rats in the paired condition (filled bars) received either 5 or 15 pairings of grape and shock at training (the 23- and 16-day-old rats, respectively), whereas the rats in the unpaired condition (open bars) received the same number of grape and shock presentations, but in an explicitly unpaired fashion. 


\section{Odor Avoidance}

The mean $( \pm S E M)$ percentage of time spent in the odor zone is depicted in Figure 4. An inspection of this figure suggests that the rats given odor-shock pairings, at either 16 or 23 days of age, avoided the odor, as compared with the rats given the odor and the shock in an explicitly unpaired fashion. Furthermore, although there was still a slight difference between the paired rats trained at 23 and 16 days of age, the magnitude of this difference appears to be much smaller than that observed in Experiment 1 (i.e., reducing the number of training trials for the 23-day-olds in this experiment seems to have been effective in reducing the level of odor avoidance). A statistical analysis confirmed these interpretations of the data. Specifically, an analysis of the odor avoidance test data revealed a significant effect of condition $[F(1,36)=46.7, p<.001]$, but the main effect of age was not significant $[F(1,36)=3.30$, $p=.08]$. The interaction of condition and age also was not significant $(F<1.0)$.

To further examine whether age differences in the amount of fear elicited by the odor contributed to the finding that the rats trained at 23 days of age exhibited the OPS effect, whereas the rats trained at 16 days of age did not, we analyzed the performance of a subset of rats from the two age groups. Specifically, we analyzed the data for the five 16-day-old rats that exhibited the strongest odor avoidance and the five 23-day-old rats that exhibited the weakest avoidance. The level of odor avoidance was extremely similar in these subsets $[M=5 \%$ vs. $7.6 \%$ time in the odor zone for the 16- and the 23-day-olds, respectively; $t(8)=$ $0.73, p=.48]$. Nonetheless, the 16-day-olds in this subset did not exhibit an OPS effect $(M=-7.8 \%)$, whereas the 23-day-olds did $[M=57.6 \% ; t(8)=3.05, p=.025]$. These data clearly do not support a threshold account of the observed developmental dissociation of conditioned odor avoidance and OPS. However, it should be noted that we have not yet examined this threshold hypothesis by giving more training to the 16-day-olds (i.e., by giving more than 15 odor-shock pairings, in an attempt to increase their odor avoidance to the level that is observed in the 23-day-olds).

\section{EXPERIMENT 3}

The results of Experiment 1 (and of Richardson et al., 2000) show that the rats trained at 16 days of age but tested at 23 days of age do not exhibit OPS even though the odorshock association is retained over the 1-week interval. In the present experiment, we examined whether the rats trained at 16 days of age would exhibit a "savings" effect if given odor-shock pairings at 22 days of age. Specifically, we had previously shown that, in adult rats, one odor-shock pairing does not produce OPS, whereas five odor-shock pairings produces an asymptotic level of OPS (Richardson, Vishney, \& Lee, 1999). Perhaps rats trained at 16 days of age would require only one odor-shock pairing at 22 days of age in order to exhibit OPS.
In the present experiment, rats from two conditions were tested. The rats in one condition (Group PT $=$ previously trained) were given 15 odor-shock pairings at 16 days of age, whereas the rats in the other condition (Group $\mathrm{N}=$ naive) were untreated. At 22 days of age, the rats from each condition were given $0,1,3$, or 5 odor-shock pairings. All the rats were tested on the following day for both OPS and odor avoidance.

\section{Method}

\section{Subjects}

Eighty experimentally naive Sprague Dawley-derived rats, from the same source and maintained as those in Experiment 1, were used. The rats were 16 days of age at the start of the experiment.

\section{Procedure}

At 16 days of age, half of the rats received 15 pairings of grape odor with shock (see the General Method section), and half were untreated (previously trained and naive conditions, respectively). At 22 days of age, all the rats were placed into the startle cage for a 2min adaptation period. Following this, a specimen jar was placed under the startle cage for 7-10 sec on five separate trials (each trial separated by $1.5,2$, or $2.5 \mathrm{~min}$ ). The jar contained $0.1 \mathrm{~mL}$ of grape on zero, one, three, or five trials (the last three trials for those given three grape exposures; the last trial for those given one grape exposure). On each trial in which grape was presented, the rats were shocked (see the General Method section). Thus, the rats in this experiment were randomly divided into eight groups (all $n \mathrm{~s}=10$ ): PT-0, PT-1, PT-3, PT-5, N-0, N-1, N-3, and N-5 (the first label refers to whether the rats had been given odor-shock pairings at 16 days of age or not, and the second label refers to the number of odor-shock pairings given at 22 days of age). All the rats were tested at 23 days of age for both OPS and odor avoidance (in a counterbalanced order). Two rats ( 1 from Group PT-1 and 1 from Group N-3) were excluded from the data analysis (the rat from Group PT-1 was excluded because its OPS performance was more than 5 SDs away from the group mean, and the rat from Group N-3 was excluded because of experimenter error).

\section{Results and Discussion}

\section{Startle}

Baseline. The mean $( \pm S E M)$ baseline startle amplitude for each group is presented in Table 3 . The effects of condition (i.e., previously trained or naive) and number of training trials at 22 days of age (i.e., zero, one, three, or five odor-shock pairings) and the interaction were all nonsignificant (all $F \mathrm{~s}<1.0)$.

Test. The mean ( $\pm S E M)$ percentage change from baseline startle amplitude during the odor test is presented in Figure 5. An inspection of this figure suggests that the rats

Table 3

Mean $( \pm S E M)$ Baseline Startle Response in Previously Trained and Previously Naive Rats in Experiment 3

\begin{tabular}{|c|c|c|c|c|c|c|c|c|}
\hline \multirow[b]{3}{*}{ Condition } & \multicolumn{8}{|c|}{ Number of Odor-Shock Pairings at 23 Days of Age } \\
\hline & \multicolumn{2}{|c|}{0} & \multicolumn{2}{|c|}{1} & \multicolumn{2}{|c|}{3} & \multicolumn{2}{|c|}{5} \\
\hline & $M$ & SEM & $M$ & SEM & $M$ & SEM & $M$ & $S E M$ \\
\hline Previously trained & 55.4 & 10.0 & 62.4 & 13.7 & 59.2 & 8.7 & 54.3 & 8.5 \\
\hline Previously naive & 49.8 & 10.7 & 54.3 & 12.9 & 44.6 & 5.6 & 54.3 & 11.0 \\
\hline
\end{tabular}




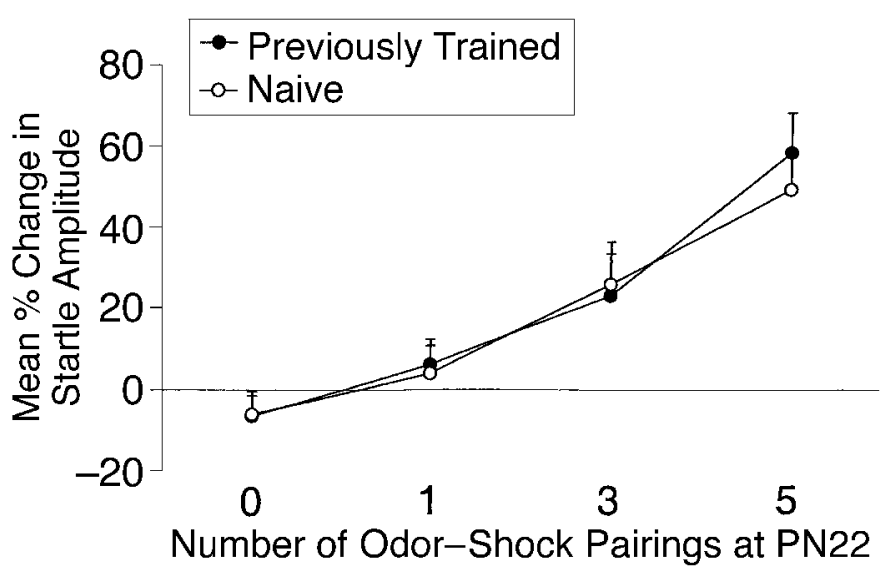

\begin{abstract}
Figure 5. Mean $( \pm S E M)$ percentage change in startle amplitude during presentation of grape odor in 23-day-old rats. The rats in the previously trained condition (filled symbols) received 15 pairings of grape odor and shock at 16 days of age, and the rats in the naive condition (open symbols) received no treatment at 16 days of age. At 22 days of age, the rats from each condition received $0,1,3$, or 5 pairings of grape odor and shock.
\end{abstract}

given 15 paired presentations of grape and shock at 16 days of age did not acquire the OPS effect any more rapidly than did the naive rats. That is, these rats did not exhibit a "savings" effect. A statistical analysis confirmed these interpretations. Specifically, an analysis of the test data yielded a significant main effect of number of training trials given at 22 days of age $[F(3,70)=21.57, p<$ $.001]$. However, the effect of previous training was not significant, nor was the interaction of these two factors significant (both $F_{\mathrm{S}}<1.0$ ). The significant effect of number of training trials was due to the gradual increase in the magnitude of the OPS effect as the number of odor-shock pairings increased. The rats receiving only a single odorshock pairing did not exhibit the OPS effect (i.e., they responded similarly to the rats given 0 odor-shock pairings). This finding supports data reported for adult rats (Richardson et al., 1999). With 5 odor-shock pairings, however, the 23-day-olds appeared to exhibit asymptotic levels of OPS (compare the performance of Group N-5 in this experiment with the performance of the 23-day-old rats in Group P1, given 15 odor-shock pairings, in Experiment 1). This finding is also in accord with previously published findings from our laboratory (Richardson et al., 2000). Those rats given 3 odor-shock pairings exhibited an intermediate level of OPS. Most important, however, the results of this experiment show that giving rats 15 odor-shock pairings at 16 days of age does not facilitate their later acquisition of the OPS effect to the same odor.

\section{Odor Avoidance}

The mean $( \pm S E M)$ percentage of time spent in the odor zone is depicted in Figure 6. An inspection of this figure suggests that (1) the previously trained rats did retain the odor-shock association over the 7-day retention interval (i.e., the previously trained rats given zero odor-shock pairings at 22 days of age still avoided the odor at test) and (2) as few as one odor-shock pairing produces a significant odor avoidance in naive rats. A statistical analysis confirmed these interpretations of the data. Specifically, an analysis of the odor avoidance test data revealed significant main effects of previous training and number of training trials given at 22 days of age [smallest $F(1,70)=$ $13.48, p<.001]$, as well as a significant interaction of these two factors $[F(3,70)=6.11, p=.001]$. The significant interaction was due to the previously trained rats' exhibiting a stronger odor avoidance than did the naive rats following either zero or one odor-shock pairing at 22 days of age [smallest $t(17)=2.74, p=.017$ ] but the rats from the two conditions' exhibiting similar levels of odor avoidance following either three or five odor-shock pairings at 22 days of age [largest $t(17)=0.19, p=.85$ ].

\section{GENERAL DISCUSSION}

Pairing of an initially neutral odor with shock leads to different conditioned responses (CRs), depending on the rat's age at training. Specifically, if the rats are youngerthan 23 days of age at training and then are tested the following day, they express the odor-shock association via odor avoidance, but not via conditioned OPS. Rats 23 days of age and older express the odor-shock association via both responses (Experiments 1, 2, and 3; see, also, Richardson et al., 2000). This developmentaldifference is not due to a threshold effect caused by the odor's eliciting different levels of learned fear at the two ages (Experiment 2). In addition, the results of the present study show that rats trained at 16 days of age but then tested at 23 days of age respond in a manner appropriate to their age at training, not their age 


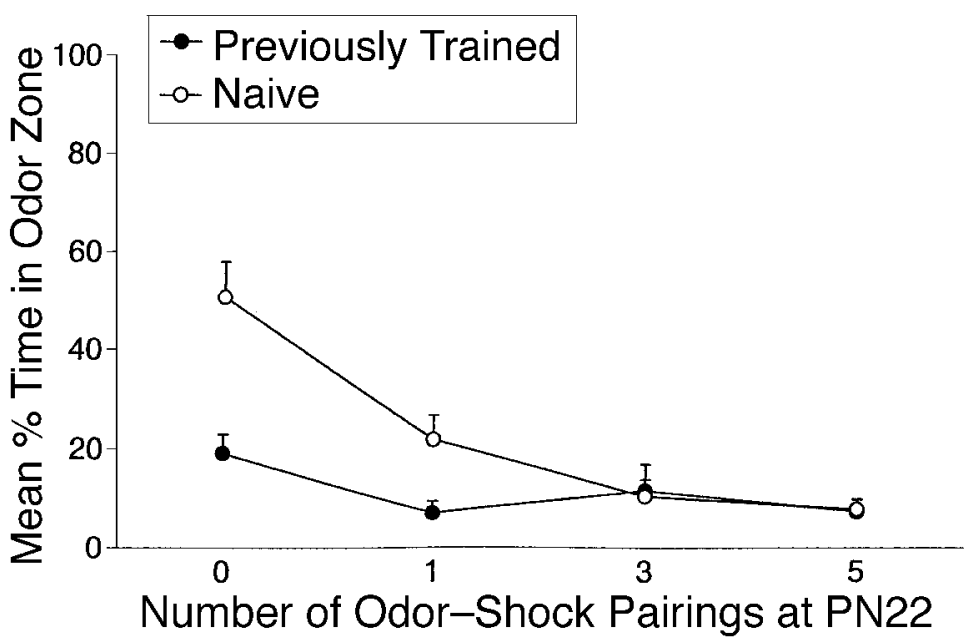

Figure 6. Mean $( \pm S E M)$ percentage of time in the odor zone during a 3-min test in 23-day-old rats. The rats in the previously trained condition (filled symbols) received 15 pairings of grape odor and shock at 16 days of age, and the rats in the naive condition (open symbols) received no treatment at 16 days of age. At 22 days of age, the rats from each condition received $0,1,3$, or 5 pairings of grape odor and shock.

at testing (Experiments 1 and 3). That is, rats in this condition express learned fear of the odor via avoidance, but not via potentiation of the startle response. Finally, the results of Experiment 3 show that rats given 15 odor-shock pairings at 16 days of age require the same number of odor-shock pairings at 22 days of age as do naive rats in order to exhibit the OPS effect. In other words, the pairing of odor and shock at 16 days of age does not produce a "savings" effect in terms of acquisition of OPS, even though these early training trials clearly lead to acquisition of an association between the odor and the shock. It should be emphasized that all the experiments in this study employed a within-subjects design; therefore, the failure to observe the OPS effect in rats trained at 16 days of age and then tested at 23 days cannot be attributed to these rats' not learning, or remembering, the CSUS association, because these rats exhibited a pronounced odor avoidance response.

From a developmental perspective, it is important to know whether animals express learned fear in a manner appropriate to their age at training or their age at testing. Although this is a difficult issue to examine empirically, there have been several previous attempts to explore this interesting, and important, issue. For example, in an examination of appetitive conditioning to an olfactory CS, Johanson and Hall (1984) reported that rats trained at one age and then tested at a later age responded in a manner appropriate to their age at testing, not their age at training. More specifically, Johanson and Hall first documented that rats of different ages exhibited a different response profile to an odor that had been previously paired with milk. Rats trained at 3 days of age exhibited a pronounced behavioral activation when exposed to the odor CS, whereas rats trained at 9 days of age exhibited a much more discrete response profile, mainly consisting of mouthing and probing responses. Importantly, Johanson and Hall reported that rats trained at 3 days of age but tested at 9 days of age exhibited discrete mouthing and probing responses when reexposed to the odor CS. Johanson and Hall concluded that "as pups grow older, specific responses may not be retained and elicited by a conditioned stimulus, though the stimulus may elicit other behaviors more appropriate to the pups' present age" (p. 154). This conclusion, clearly, is quite different from that reached in the present study.

There are a number of possible explanations for this apparent discrepancy. For example, although both studies involved olfactory conditioning in young rats, the study by Johanson and Hall (1984) used appetitive conditioning procedures, whereas our study used aversive conditioning procedures. Furthermore, we used slightly older rat pups than those used by Johanson and Hall. It is also possible, as was acknowledged by Johanson and Hall, that their results reflected differential forgetting rates for different CRs. In other words, although the response profile of the 3-day-old rats was dominated by intense, diffuse behavioral activation, these animals did exhibit some mouthing and probing behavior. Given this, it is possible that the observed responses in the rats trained at 3 days of age and tested at 9 days of age were a consequence of the rats' retaining the mouthing and probing components of the acquired CR but forgetting the diffuse behavioral activation component of the CR. As was recognized by Johanson and Hall, the best way to resolve this interpretative problem would be to have a situation in which rats are trained at an age at which they definitely do not express a particular CR 
but then are tested at an age at which they clearly can express that response. That is exactly what we have achieved in the present study.

The findings of the present study also appear to be in contrast to a recent analysis of eyeblink conditioning in the developing rat. Specifically, it has been shown that rats given pairings of an auditory CS and shock exhibit conditioned eyeblinks to that CS if training takes place at 24 days of age, but not if training occurs at 17 days of age. Rats in the younger age group do acquire the CS-shock association, since they exhibit conditioned freezing when reexposed to the CS (see Stanton, 2000). Stanton, Fox, and Carter (1998) suggested that the lack of conditioned eyeblinks in the 17day-old rat is a problem of expression, rather than a problem in acquisition. That is, Stanton et al. reported that giving rats CS-shock pairings at 17 days of age led to a "savings" effect when those rats were given CS-shock pairings at 24 days of age. This is clearly not in agreement with the results of Experiment 3 in the present study.

This apparent discrepancy between the findings of Stanton et al. (1998) and those of the present study are particularly surprising given the numerous similarities between the two conditioning procedures used. That is, both the eyeblink-conditioning procedure and the potentiationof-startle procedure involve aversive conditioning, and both responses emerge relatively late in development (i.e., around 23-24 days of age). Furthermore, both involve increased responsiveness of a basic reflex mediated by brain stem structures. Nevertheless, it appears that these two model preparations differ markedly in terms of the effects of early conditioning experience on subsequent performance of the response following later training. Whereas giving young rats CS-US pairings at an age at which they cannot exhibit conditioned eyeblink responses appears to lead to faster acquisition of the eyeblink response following training at a later age (Stanton et al., 1998), doing the same thing does not lead to faster acquisition of the OPS effect (Experiment 3 of the present study). Another apparent developmental difference between these two model preparations concerns the effects of early nonreinforced exposure to the CS. Specifically, it appears that preexposing rats to the CS at an age at which they do not exhibit conditioned eyeblink responses actually enhances conditioning to that CS (see Stanton, 2000), but we do not find this effect in the OPS procedure (Richardson, Fan, \& Parnas, in press). It will be important to more fully examine those situations in which eyeblink conditioning and fear potentiation of startle differ during development.

Although the results of the present study appear to contradict several findings in the literature, they are supported by several other recent findings. For example, we have previously reported similar findings from a betweengroup design (Richardson et al., 2000). Furthermore, Sullivan, Landers, Yeaman, and Wilson (2000) have reported findings that are consistent with those reported in the present paper. Specifically, Sullivan et al. showed that rats given odor-shock pairings prior to Postnatal Day (PN) 10 exhibit a preference for that odor, whereas rats given the same odor-shock pairings after PN10 avoid that odor. Im- portantly, Sullivan et al. reported that rats trained prior to PN10 but tested at an older age continued to exhibit a preference for the odor. In other words, these rats responded in a manner appropriate to their age of training, not their age of testing. One could interpret these findings as being due to the PN10-day-old rats' encoding the odor as predicting something "good" and the older rats' encoding it as predicting something "bad." Thus, when rats trained at PN10 are tested at an older age, the odor CS activates an expectation of something "good," and the animal therefore expresses a preference for the odor CS. Such an interpretation (i.e., one focusing on the nature of how the US is encoded at training) cannot be offered for the findings of the present study.

The literature reviewed above illustrates two important points. First, there is very little empirical evidence concerning the issue of whether animals respond to a CS in a manner appropriate to their age at training or their age at testing. Second, there are some striking, apparent differences in this small literature: Some investigators have reported that rats respond in a manner appropriate to their age of training, whereas others have reported that they respond in a manner appropriate to their age at testing. Clearly, much more research is needed not only to resolve these apparent discrepancies, but also to increase our understanding of this fundamental developmental issue.

Finally, the results of the present study seem to raise some serious issues for the currently dominant view of the emotive state elicited by an aversive CS. Most of the current views of learned fear postulate that an aversive CS elicits a central state of fear that is then expressed via any of a number of behavioral responses (e.g., Davis, 1992). From this perspective, rats trained at 16 days of age but then tested at 23 days of age would have been expected to show both odor avoidance and the conditioned OPS effect, assuming, of course, that they retained the odor-shock association over the 1-week interval. Clearly, this is not what happened in the present study. Therefore, we would like to suggest that CS-US pairings produce neural plasticity not only in the amygdala, as is widely accepted, but also in structures downstream from the amygdala (i.e., the various structures to which the amygdala projects and that mediate specific behavioral responses indicative of learned fear). This is not an entirely original proposal, in that others have also suggested some type of multiple-memory system (e.g., Stanton, 2000). In any case, we propose that if the projection from the amygdala to a downstream structure is not fully functional (e.g., because of the animal's immaturity at the time of training), the neural plasticity required for later behavioral expression of learning will not occur in that structure. Thus, when the animal is tested at a later age, the specific behavioral manifestation of learning mediated by that downstream structure will not be exhibited. This idea illustrates the potential value of a developmental approach to the analysis of the neural bases of learned fear.

Our proposal can be tested in a number of ways. For example, it is based on the assumption that the projection between the amygdala and the PnC (the brainstem struc- 
ture that mediates conditioned fear potentiation of startle) is not functional in rats younger than 23 days of age. This assumption is based on two pieces of evidence. First, given that rats younger than 23 days of age reliably exhibit learned fear when measures such as freezing are recorded (see Hunt \& Campbell, 1997, for a review), there is no doubt that the amygdala undergoes the necessary neural plasticity for exhibiting learned fear. Second, recent evidence has shown that neural activity in the primary startle pathway can be pharmacologically increased, thus leading to larger startle responses in rats as young as 16 days of age (the youngest age tested; Weber \& Richardson, 2001). Taken together, this shows that all the pieces required for conditioned fear potentiation of startle being observed are present in rats younger than 23 days of age; however, they simply do not exhibit the effect. As was noted, our interpretation of the failure to observe conditioned fear potentiation of startle in rats younger than 23 days of age is that the pathway from the amygdala to the $\mathrm{PnC}$ is not functional prior to that. Therefore, the neural plasticity required for expressing fear potentiation of startle does not occur in the $\mathrm{PnC}$ of rats trained prior to 23 days of age. However, our basic assumption has not yet been tested directly. That is, we do not yet know whether the pathway between the amygdala and the PnC is truly not functional prior to 23 days of age. Given the evidence that direct electrical stimulation of the amygdala leads to potentiated startle responses in adult rats (Rosen \& Davis, 1988), it will be relatively straightforward to test this assumption in future research.

A second way of testing our proposal is to temporarily inactivate various downstream structures from the amygdala during training of adult rats. For example, do adult rats that have their PnC inactivated during training express odor-elicited freezing, but not OPS? We are currently exploring this possibility. If the findings are comparable to what has been seen with the developing rat (the present study), this would suggest that some modifications are needed to current theoretical formulations of learned fear. If the findings do not conform to what has been seen in the developing rat, this would suggest that young and adult rats are acquiring different types of CS-US associations (e.g., stimulus-stimulus vs. stimulus-response). Either outcome will be important in increasing our understanding of learning and memory mechanisms, from both a developmental and a neural perspective.

\section{REFERENCES}

CArrive, P. (2000). Conditioned fear to environmental context: Cardiovascular and behavioural components in the rat. Brain Research, 858, 440-445.

DAvis, M. (1992). The role of the amygdala in fear and anxiety. Annual Review of Neuroscience, 15, 353-375.
FANSELOW, M. S. (1990). Factors governing one-trial contextual conditioning. Animal Learning \& Behavior, 18, 264-270.

Hunt, P. S., \& CAMPBell, B. A. (1997). Developmental dissociation of the components of conditioned fear. In M. E. Bouton \& M. S. Fanselow (Eds.), The functionalbehaviorism of Robert C. Bolles: Learning, motivation, and cognition (pp. 53-74). Washington, DC: American Psychological Association.

Hunt, P. S., Richardson, R., \& Campbell, B. A. (1994). Delayed development of fear-potentiated startle. Behavioral Neuroscience, 108, 69-80.

Hunt, P. S., Richardson, R, Hess, M. F., \& Campbell, B. A. (1997). Emergence of conditioned cardiac responses to an olfactory CS paired with an acoustic startle UCS during development: Form and autonomic origins. Developmental Psychobiology, 30, 151-163.

JoHANSON, I. B., \& HALL, W. G. (1984). Ontogeny of appetitive learning: Independent ingestion as a model motivational system. In R. Kail \& N. E. Spear (Eds.), Comparative perspectives on the development of memory (pp. 135-157). Hillsdale, NJ: Erlbaum.

McNish, K. A., Gewirtz, J. C., \& Davis, M. (1997). Evidence of contextual fear after lesions of the hippocampus: A disruption of freezing but not fear-potentiated startle. Journal of Neuroscience, 17, 9353 9367.

Richardson, R., Fan, M., \& Parnas, A. S. (in press). Latent inhibition of conditioned odor potentiation of startle: A developmental analysis. Developmental Psychobiology.

Richardson, R., Paxinos, G., \& Lee, J. (2000). The ontogeny of conditioned odor potentiation of startle. Behavioral Neuroscience, 114, 1167-1173

Richardson, R., Tronson, N., Bailey, G. K., \& Parnas, A. S. (2002). Extinction of conditioned odor potentiation of startle. Neurobiologyof Learning \& Memory, 78, 426-440.

Richardson, R., Vishney, A., \& Lee, J. (1999). Conditioned odor potentiation of startle. Behavioral Neuroscience, 113, 787-794.

Rosen, J. B., \& DAVIS, M. (1988). Enhancement of acoustic startle by electrical stimulation of the amygdala. Behavioral Neuroscience, 102, 195-202.

Rudy, J. W., \& Cheatle, M. D. (1977). Odor-aversion learning in neonatal rats. Science, 198, 845-846.

Sananes, C. B., Gaddy, J. R. \& CAmpbell, B. A. (1988). Ontogeny of conditioned heart rate to an olfactory stimulus. Developmental Psychobiology, 21, 117-133.

Stanton, M. E. (2000). Multiple memory systems, development and conditioning. Behavioural Brain Research, 110, 25-37.

Stanton, M. E., Fox, G., \& Carter, C. S. (1998). Ontogeny of the conditioned eyeblink response in rats: Acquisition or expression? Neuropharmacology, 37, 623-632.

Sullivan, R. M., Landers, M., Yeaman, B., \& Wilson, D. A. ( 2000). Good memories of bad events in infancy. Nature, 407, 38-39.

Vishney, A., \& Richardson, R. (2000). Diazepam attenuates conditioned odor potentiation of startle in rats. Psychobiology, 28, 515-519.

Weber, M., \& Richardson, R. (2001). Centrally administered corticotropin-releasing hormone and peripheral injections of strychnine hydrochloride potentiate the acoustic startle response in preweanling rats. Behavioral Neuroscience, 115, 1273-1282.

Westbrook, R. F., Good, A. J., \& Kiernan, M. J. (1994). Effects of the interval between exposure to a novel environment and the occurrence of shock on the freezing responses of rats. Quarterly Journal of Experimental Psychology, 47B, 427-446.

(Manuscript received June 6, 2002; accepted for publication July 25, 2002.) 